\title{
A qualitative study of pediatric residents' developmental oral case presentations
}

\author{
Angela Orsino ${ }^{1}$, Lesley Gotlib Conn ${ }^{1}$, Anne Kawamura ${ }^{1}$, Lorelei Lingard ${ }^{2}$ \\ ${ }^{1}$ Child Development Program, Holland Bloorview Kids Rehabilitation Hospital, 150 Kilgour Rd, Canada \\ ${ }^{2}$ Department of Medicine, Schulich School of Medicine and Dentistry, University of Western Ontario, Canada
}

Correspondence: Angela Orsino, Child Development Program, Holland Bloorview Kids Rehabilitation Hospital, 150 Kilgour Rd, Toronto, Ontario, Canada, M4G 1R8. Email: aorsino@hollandbloorview.ca

\begin{abstract}
Objectives: To explore how pediatric residents apply their knowledge of relevance in their oral case presentations to the unfamiliar context of a chronic care setting, that of developmental pediatrics.

Methods: Pediatric resident oral case presentations were observed and semi-structured interviews were conducted with residents and staff developmental pediatricians. A total of 13 residents and 5 developmental pediatricians were observed and 13/18 participants were interviewed and audiorecorded. Qualitative transcript data were analyzed for emergent themes using an inductive thematic analysis approach.

Results: Pediatric residents identified challenges with determining relevance in their oral case presentations in this context. They had difficulty generating succinct case presentations and integrating patient information into an overall clinical impression. Residents conflated the strict
\end{abstract}

organization of information with relevance, and demonstrated presentation formats akin to those used by more novice medical trainees. Staff members viewed the oral case presentation as a flexible vehicle to integrate patient information; however, this perspective was not always explicitly shared during teaching exchanges.

Conclusions: Results of this study draw attention to the possible contextual nature of residents' understanding and use of relevance principles in their oral case presentations. Clinicians responsible for teaching in outpatient chronic care settings need to be aware of the possible challenges residents may have with their case presentation, so that teaching strategies can be implemented to ensure residents' acquisition and appropriate adaptation of this essential communication skill.

Keywords: Oral case presentation, developmental paediatrics, relevance

\section{Introduction}

The oral case presentation (OCP) plays an essential role in the medical care of patients. Through the OCP, health care professionals communicate important information about patients. In an effective OCP, a patient's story is filtered, reorganized and delivered in a concise manner to generate a diagnostic impression and appropriate treatment plan. In a clinical teaching setting, the OCP delivered by medical trainees not only serves to support patient care but also plays a pivotal role in medical education. ${ }^{1}$

A previous study of medical students' OCPs indicates that novice trainees struggle with the concept of relevance. ${ }^{2}$ Understanding the concept of relevance enables the physician-communicator to trim away excess information to create a concise discourse in medicine. ${ }^{2}$ Key to the concept of relevance are aspects of both clinical and rhetorical knowledge. Clinical knowledge is specific to the patient's clinical course. Rhetorical knowledge, on the other hand, is context-based and specific to the intended audience and the purpose of their discourse. ${ }^{2}$ Application of both clinical and rhetorical relevance principles are important in composing an effective OCP.

Both trainees and teachers highlight the importance of using relevance principles to help determine what information to include in an OCP and also acknowledge that this concept is difficult to teach and learn. ${ }^{2}$ Previous studies have shown that novice presenters (medical students) lack rhetorical expertise ${ }^{2,3}$ and have difficulty in determining what and how much information to 
include/exclude in their OCPs. They have particular difficulty with this when confronted with complex medical cases. $^{1-3}$ These novice clinicians have been described as using the OCP as "a rigid, rule-based data storage activity governed by order and structure". Teachers, on the other hand view the OCP as a "flexible means of communication" 3 and a means to "make a case" for the assessment and plan. $^{4}$

Through the OCP, medical teachers can also obtain insight into a learner's understanding of a patient's problem and their clinical reasoning processes. Through teaching and feedback around the OCP, medical teachers are in the optimal position to correct trainees' misperceptions and foster learning of effective OCP skills. Messages conveyed to students can shape their speaking, thinking, perceiving and behaving as future physicians. ${ }^{1}$ Previous studies, however, have shown that faculty feedback to students around OCPs contains mostly tacit messages which can be misinterpreted by trainees and lead to inappropriate value acquisition and dysfunctional generalizations. ${ }^{2,3} \mathrm{~A}$ recent study showed that staff interruptions during junior resident internal medicine OCPs resulted in detours being taken in the OCP and that these were essential in facilitating teaching. ${ }^{5}$ However, similar to previous studies, staff failed to explicitly indicate what strategy they were using in reviewing the case, leaving trainees to interpret this on their own. Furthermore, Holmboe ${ }^{6}$ described that staff are uncomfortable and insufficiently prepared to give feedback to residents on their clinical performance.

\section{Rationale for current study}

Existing OCP research centers mostly on novice trainees such as medical students and information on how medical residents deal with contextual challenges in their OCP is lacking. Medical residents, as opposed to medical students, have had greater opportunity in their clinical training to practice delivery of the OCP as well as adapting their OCP to different clinical contexts. As practitioners who are on their way to independent clinical practice, medical residents are expected to master OCP skills in their postgraduate training years. Several medical training governing bodies have endorsed the necessity of developing competency in OCP skills. In Canada, the Royal College of Physicians and Surgeons of Canada has outlined core clinical competency skills (CanMEDs roles) expected to be achieved by medical trainees. $^{7}$ Under this CanMEDs framework, OCP skill development is viewed as an essential element in helping to fulfil both "Communicator" and "Medical Expert" roles, two of the seven core competency domains outlined by this organization. Similarly, the Accreditation Council of Graduate Medical Education and the American Academy of Pediatrics published learning expectations for pediatric residents. ${ }^{8}$ In this document, the OCP is described as a form of interprofessional communication which is a critical skill to achieve in medical practice. It also highlights the need for trainees to develop their rhetorical knowledge to be able to adapt the message to the relevant context (the audience, the purpose and occasion). They outline a continuum in which trainees are expected to progress from, at a minimal level, the ability to recite facts in a rigid rule-based format, often from a template, not context-specific, to become a "master in improvisation", "tailoring the message to context to gain maximum effect."

Given the expectations of residents around OCP skill development, it is important to understand how these more experienced trainees apply their knowledge of relevance to their OCPs, particularly with respect to the more challenging rhetorical aspects. Understanding what challenges residents confront in their OCPs will inform development of more targeted and effective teaching strategies of this necessary skill.

The relatively greater experience of residents in conducting OCPs in varying clinical rotations suggests they would have better developed OCP skills versus medical students. Haber and Lingard's study ${ }^{3}$ supports this as they found that internal medicine residents versus medical students were better able to adapt their OCPs to different acute care settings. The finding by Yang et $\mathrm{al}^{9}$ that the frequency of staff interruptions during emergency room OCPs is inversely correlated with level of trainee experience can also be interpreted to suggest a similar concept. Results from other studies are, however, conflicting. Papp and Wolpaw $^{10}$ compared clinical reasoning skills in the OCP in third versus first year internal medicine residents in an outpatient setting and found no significant difference in these two cohorts. They also found that overall, $80 \%$ of the presentation time was spent conveying facts, highlighting the need for residents to learn OCP presentation skills that also communicate clinical reasoning. Another study conducted in general medical clinics found residents did not consistently report information such as psychosocial content, their assessment, or plan in their OCPs. ${ }^{11}$

It is clear from these studies that further understanding of how residents use their rhetorical knowledge skills to adapt their OCPs in different clinical contexts is necessary. One contextual challenge residents confront is the shift between clinical settings in terms of managing acute versus chronic issues. Patients with chronic health issues tend to be medically complex, with both acute medical comorbidities and psychosocial issues. Residents are challenged in this type of OCP to extend their focus beyond acute issues, which may have readily available medical interventions, to encompass broader issues impacting on patients' lives.

In the pediatrics realm, the outpatient developmental pediatrics clinic is one such chronic care setting. Children assessed and followed in these clinics have chronic medical and developmental concerns. Pediatric residents in this clinical setting are confronted with a unique set of contextual challenges as compared to their acute care clinical rotations. As such, the developmental pediatric outpatient 
clinic provides an optimal setting for studying how residents use their understanding of relevance to compose OCPs.

The objective of this study was to evaluate how second year pediatric residents extrapolate their knowledge of relevance in their OCP to cases with chronic and complex medical and psychosocial needs.

\section{Method}

\section{Study design}

The study was conducted at an academic tertiary care outpatient clinic for children with physical disabilities and developmental disorders. Pediatric residents spend four weeks in this setting during the second year of their residency training program. They conduct new and follow-up patient assessments for children with neurodevelopmental diagnoses such as autism spectrum disorder, cerebral palsy and developmental disability. Patients frequently have multiple medical issues (eg. genetic conditions, hearing and visual impairment, seizures) in addition to developmental and psychosocial needs. Following all assessments, residents review the case with a staff developmental pediatrician in a small meeting room within the clinic separate from patients and families. During their rotation, residents receive didactic teaching sessions on relevant topics related to developmental pediatrics. They also obtain an orientation package with relevant background reading material and a history-taking template (developed within our department) to help facilitate acquisition of more detailed developmental information on history.

The length of the OCP varies according to the nature of the appointment and complexity of the patient concerns. During this study, OCPs based on new patient assessments lasted between 20 and 60 minutes. Those based on followup patients averaged 20 minutes.

A qualitative analysis of observations of resident OCPs was conducted in this study. Research ethics approval was obtained by the hospital's ethics review board prior to the study.

\section{Participants}

A convenience sample of 13 second year pediatric residents and five staff developmental pediatricians was recruited. No study participants declined participation in the study. Written consent was obtained from participants before observations were conducted.

\section{Study procedure and data collection}

Data collection was designed in two phases:

\section{Field Observations}

Twenty two OCPs were observed and audio-recorded by either the primary author (AO) or research assistant (LGC). All observed OCPs were eligible for inclusion. There were no exclusion criteria. Each resident-participant was recorded at least once, up to a maximum of four times. Each staff was recorded between two and nine times throughout the study period.

\section{Interviews}

Two researchers (AO, LGC) conducted and audiorecorded semi-structured interviews with eight residents at the end of their rotations (the remaining five were not available for interviews). The interview questions were designed to elicit their perspectives on the challenging aspects of the developmental OCPs, the approach they used to determine relevance in this setting, and their experience with staff feedback. Similarly, at the end of the data collection period we interviewed all staff regarding their perspectives on relevance in the OCP, their opinions on residents' determination of relevance, and how they delivered feedback during the OCP.

\section{Data analysis}

Data were analyzed using an inductive thematic analysis approach $^{12}$ to find recurrent themes in the data set. Transcripts of observed OCPs were read independently by the same two researchers conducting the observations. A coding structure of recurrent themes was established. Data were collected to the point of saturation, that is, when the ongoing analysis of new data ceased to produce any new insights relevant to the emerging themes. ${ }^{13}$ Transcribed interview data were subsequently used to triangulate and validate findings from observational data.

\section{Results}

The following themes were derived from the data set:

\section{Not a typical OCP}

Residents identified unique challenges with the OCP not encountered in acute care clinical rotations, stating that

"the developmental case presentations are a little bit different than other areas of pediatrics"[Resident \# 11 interview].

Residents perceived that the developmental history was not truly "medical." They described struggling with the complexity of developmental cases, which often had acute medical issues enmeshed with chronic ones. Residents also felt they had limited background knowledge about certain developmental conditions and lacked experience dealing with them. As one resident explained,

"It's more challenging in this rotation to determine what's relevant because of the unfamiliarity with the core material." [Resident \# 1 interview] 


\section{Organization}

Residents identified the concept of organization as a critical component in deciphering relevant information. Some equated being organized with relevance in their OCP. Resident \#11 found the developmental OCP challenging because "sometimes, I would have trouble with trying to organize my ideas and how exactly to present it." In response to the interviewer trying to clarify whether she had difficulty determining what information was relevant in the OCP, she responded:

"Um, maybe...I feel like it's more organizational. Um, like what's relevant. Yeah, I guess that goes into organization, because the relevant things should be presented first and then the still relevant but less so should be presented second, I guess"

As a result of conflating organization and relevance, residents' developmental OCPs tended to be presented in inflexible formats, relaying information under separate categories or subheadings. Residents demonstrated a need to abide by certain perceived rules in delivering their OCP whereby information was compartmentalized into appropriate headings. One resident commented on his awareness and uncertainty of the rules of the developmental OCP by commenting to a staff member:

"I don't even know if toe walking goes into gross motor... I just threw it in there." [Observation \# 20, Resident \# 12]

Residents also sought guidance from staff about what order they preferred to hear information in the OCP, suggesting that organization was perceived to be as important as relevance, as depicted in the following excerpt:

Resident: I'll do school [history] with social, that probably would make more sense. Um, would you like the medical and diet history, that stuff, now?

Staff: Sure

Resident: Or do you want me to do all the social development stuff? How do you like it?

Staff: I don't mind, whatever way is comfortable for you. Resident: Okay. The medical history is fairly brief anyways. Staff: Sure. [Observation \# 20, Resident \# 12]

Residents' inflexibility in the OCP was demonstrated by their reluctance to answer questions posed by staff members which did not relate concretely to the category being discussed by the resident at that time. The following excerpt depicts how a resident deferred discussing information that crossed such boundaries in her presentation:

Resident: They were first concerned in her first year. She was late to develop her milestones and she had speech regression at two years of age.

Staff: How much had she said before the regression?

Resident: Um, I'll go into a lot of details for the social...

Staff: Okay, that's fine. Whenever. [Observation \# 22, Resident \# 13]
Residents also identified apprehension with respect to the rules, order and organization of their OCP when disrupted by staff members. One resident commented:

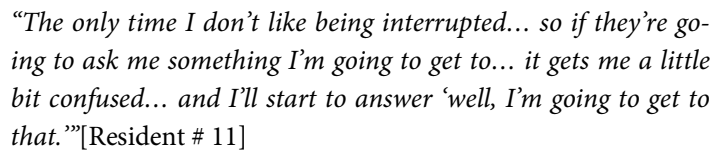

\section{Succinctness}

Residents identified succinctness as a significant challenge and important contributor to determining relevance in the OCP. Some described feeling compelled to convey all the details obtained in the history in their OCP so as not to miss any relevant information. Residents believed that in the developmental OCP there was "a lot of relevant information...because development is all encompassing" [Resident \# 13 interview]. They described how it was important in this setting versus others to obtain a more thorough developmental history, and their needing "to get every detail” [Resident \# 12 interview]. Residents' detail-oriented approach led to their perception that more information is better, and that all collected information should be presented. Some had difficulty prioritizing what information to include and how to summarize the most salient parts of the history succinctly. When asked what is considered relevant in the developmental OCP, one resident responded:

“... that very precise developmental history at every stage... take the most detailed history at every stage and it will all probably be relevant. ..." [Resident \#1]

\section{Difficulty integrating the developmental history into the "whole picture"}

Compartmentalization of information into discrete categories in the OCP resulted in residents expressing difficulty managing information that overlapped developmental categories or domains. One resident described the challenge with presenting information that crossed the boundaries of these categories:

\footnotetext{
"So things that will fall into your development history can still be part of the presenting complaint. Or things that fall into the social history, school history, can still be part of the presenting [complaint]. So that, sometimes, I would have trouble with." [Resident \# 11]
}

Residents' difficulty crossing perceived boundaries in their OCP generally led to presentations that lacked integration of information into the whole clinical picture. Many had difficulty grappling with the concept that developmental domains cannot only overlap, but also interact to result in a child's current presentation and level of functioning (for example, a child who has delayed language skills may also demonstrate delays or impairment in social functioning with their peers as a result of this). Residents prioritized 
organizing information, focusing on order and structure of content, versus processing the collected information into a coherent story that described the overall picture of the case at hand.

Residents presented an abundance of unfiltered information in their OCP which led to several difficulties. Often they presented information because it was listed on their history-taking tool, without understanding its meaning, how to integrate it into the context of the child's whole clinical/developmental picture or how the information was relevant to certain developmental disorders. The following excerpt discussing a child with autism spectrum disorder demonstrates how a resident asked about use of neologisms without knowing what the term meant:

Resident: He makes good eye contact when he speaks and he does use gestures to express himself, and he points to things that he's interested in or wants and there's no abnormal noises, um, such as echoes, and pronouns, and neo...(trying to pronounce word)

Staff: neologisms \{laughs\} ... do you know what that is? Resident: $\{$ laughs\} No. [Observation \# 16, Resident \# 12]

\section{Staff members' perspectives}

Staff and resident perspectives of relevance shared some common themes. Staff members highlighted the importance of a thorough developmental history, organization and succinctness in residents' OCPs. However differences in perspectives were also found. Staff members described a more streamlined approach to organization in which residents are able to "glean the key points under each heading," and "find the balance between giving the information back and not having to give every example that the parent described in every detail" [Staff \#3]. They clearly valued information which was filtered and summarized and included only "the pertinent things" [Staff \#2]. Residents' perception that more information was necessary to present in their OCP was not supported by staff members.

Staff members also viewed the OCP as being flexible and adaptable to the particular case. They allowed residents to organize and synthesize information as they saw fit to be able to generate a coherent story for the patient. One staff commented:

"I usually find they [residents] have their own way of being organized. It doesn't have to be the way I organize it but then you can see that they're thinking through it and making sure that they've included everything." [Staff \# 4]

Instead of a data organization tool, staff described the OCP as a means to process and integrate information with an end goal of formulating a diagnostic impression and description of a child's level of developmental functioning. Understanding the child's whole picture through the OCP was deemed highly relevant by staff. As well, interaction and overlap of developmental information was considered more relevant than compartmentalizing. One staff member's [Staff \# 4] comment regarding relevant aspects of the OCP highlighted "having enough information about the key areas of development and how the key areas interact with each other in order to lead the child and the family around the child to the point where they are presenting." Staff raised concerns about residents focusing on dividing information into "boxes" and missing the bigger picture. This same staff member identified the need for more explicit teaching with residents around this during the OCP:

"So really when we have a resident with us we should be spending far more time in observing them actually doing the interview, listening to the case presentation, and then going over with them how to integrate that and present it better."

Despite the differences in opinions on relevance in the OCP, staff member's perspectives were rarely conveyed explicitly to residents during feedback sessions.

\section{Discussion}

The findings from this study demonstrate that pediatric residents have difficulty extrapolating their relevance skills in OCPs to the context of the chronic care setting of developmental pediatrics. Residents recognize that this type of OCP is different from what they are accustomed to in acute care settings. These "expert" case presenters adopted presentation styles reminiscent of those used by more novice trainees. ${ }^{2,3}$ Residents generally reverted to using rigid, rule-bound presentation formats, emphasizing organization of information into discrete categories and had difficulty integrating and synthesizing information into a comprehensive picture or story. Residents' adherence to rigid presentation styles, for example, resisting being interrupted by staff during their OCPs, may have led to lost teaching moments, as Aziz et al. ${ }^{5}$ suggest that such "detours" provided by staff interruptions are important in teaching. Residents also demonstrated difficulty with succinctness, leading to presentation of an abundant amount of unfiltered information. Residents lost their discriminating lens for relevant data and believed that organization of masses of unfiltered information translated into relevance. Similar to medical students, distinction between clinical and rhetorical facets of relevance was not clearly demonstrated in resident OCPs in this setting.

There are several reasons why residents may have difficulty in applying their relevance skills in their OCPs to these chronic and medically complex cases. First is the acknowledgement by residents of their relative lack of background knowledge of developmental disorders. This introduces an element of uncertainty in the OCP that residents must deal with. ${ }^{14}$ Lingard et al. ${ }^{15}$ studied the rhetoric of uncertainty in medical students' OCPs and found that medical students viewed this as unacceptable, resulting in manifestation of various strategies to deal with their knowledge limitations. Reverting to more novice methods of presentation may be one strategy used by 
residents to cope with the uncertainty around their knowledge limitations in this area.

Another possible contributing factor is the element of ambiguity associated with the clinical domain of developmental pediatrics. Ambiguity is related to uncertainty and both are contributed to by the "gray areas of medicine" ${ }^{16}$ There are several characteristics of pediatric developmental cases which may appear ambiguous to residents and add to their difficulty in extracting contextually-relevant information from their cases. For example, the diagnosis of several pediatric developmental disorders does not involve a black and white, all-or-none approach. Often, disorders are on a spectrum of severity and have different clinical manifestations at various developmental levels. Developmental disorders are also subject to influence from various sources (e.g. family, psychosocial, environmental factors) and interact with acute medical issues. The amount of information gathered in this setting is often greater than in acute care and is not able to be dissected neatly into discrete categories due to the interrelatedness of developmental domains. For these reasons, patients in this setting may also be viewed as more "medically-complex." Results of previous studies have shown that increasing medical complexity of cases poses a challenge to medical students' ability to organize and filter relevant information ${ }^{1}$, a phenomenon which may be reflected in residents in this study as well.

Development of clinical reasoning and rhetorical relevance skills are inter-related as the ability to siphon out contextually-relevant information is important to the clinical reasoning process. ${ }^{17}$ In this respect, the OCP may act as a proxy for estimating a trainee's level of clinical reasoning ${ }^{18-20}$, a skill which is essential in future practice as an independent clinician. Residents' application of more novice presentation strategies and difficulty in using relevance principles effectively to generate a coherent, concise clinical story may have implications for residents' higher learning skills in clinical practice. Pangaro ${ }^{21}$ described a framework which outlines the developmental progression of trainees along a continuum of performance demonstrated from medical school through residency. He described trainees as progressing from being Reporters, to Interpreters, to Managers, to Educators (RIME) at various levels in their training. Reporter skills should be mastered in later medical school years. The transition to Interpreter "requires a higher level of knowledge, and more skill in selecting the clinical findings that support possible diagnoses"; this is the benchmark performance expectation at the residency level. The Manager-Educator roles are closely linked, requiring increasingly higher level knowledge, judgement and planning skills as well as the ability to share their knowledge and learning with others. Results from this study showed that when confronted with contextual challenges in the chronic care setting, pediatric residents demonstrate greater comfort with the Reporter versus Interpreter role.
Results of this study also showed that staff members and residents expressed dichotomous perspectives on relevance in the OCP. Similar to what has been described in the literature ${ }^{2,3,22}$ staff members in this study viewed the OCP as a vehicle to capture the overall picture of the patient, while residents were more inclined to use the OCP as a data organization tool. This discrepant viewpoint was, however, not often explicitly translated to residents during their OCP feedback. Such observed paucity of feedback to residents on how to improve their OCPs in this study is in keeping with results of previous studies. Papp and Wolpaw ${ }^{10}$ found that faculty feedback to residents during their OCPs focused mainly on eliciting and clarifying facts of the case regardless of the trainee's level of training. Jackson et al. ${ }^{23}$ reported that faculty rarely challenged medical trainees to analyze, synthesize or apply their knowledge in their OCPs and their feedback did not provide trainees with suggestions on how to improve their OCP skills. Lack of explicit feedback to medical students has been shown to potentially result in the adoption of inaccurate beliefs and assumptions. ${ }^{2}$ In this study, it is plausible that the paucity of staff members' explicit feedback may have contributed to residents' inaccurate beliefs, for example, that more is better (that all collected information is relevant in the OCP and that staff members wanted to hear more information relative to other clinical rotations).

Several strategies may be helpful in facilitating residents' understanding of relevance as it applies to the OCP. Diagnosing the learner's knowledge level (at general and specific levels) so that gaps and misperceptions can be identified and corrected is one possible strategy. This is in keeping with Irby's findings $s^{24}$ that identifying the educational knowledge of learners is an essential element of teaching excellence in clinical rounds. Also, providing residents with core content background knowledge about less familiar clinical conditions may potentially lead to less uncertainty related to these conditions. It may also provide them with the critical framework to apply their higher level critical reasoning skills. Explicit teaching of clinical reasoning skills to trainees has been shown to improve OCP skills ${ }^{25}$ and may also prove beneficial in such chronic care settings to help overcome residents' challenge with relevance. It was clear from this study that simply providing residents with a lengthy, "catch-all," history-taking template was not an effective mechanism to help residents filter, prioritize and synthesize relevant information. Residents require further guidance in using this tool in a contextually-sensitive manner.

More explicit feedback from teachers addressing gaps in perceptions of the role of the OCP, teaching of relevance concepts specific to each case (explanations of what information is relevant to include/exclude in each particular case and why) and how to summarize information into a coherent, succinct impression that captures the patient's "whole 
picture" are also plausible teaching strategies to try to actively teach relevance principles. Studies by Green et al., 22 also describe that faculty providing clear expectations for students' OCPs may also help facilitate better delivery of OCPs. Despite this clear need for effective faculty feedback to residents on their OCP skills, some studies ${ }^{6,26}$ indicate that medical educators struggle with this, suggesting that in some situations, some further faculty development training in delivering effective feedback to trainees may be necessary to accomplish this goal. The influence of these strategies on residents' knowledge and performance of relevance in the OCP in chronic care clinical settings could be explored by future studies.

The challenges confronted by pediatric residents in this setting may have broader implications and be generalisable to other outpatient medical settings where medically complex or chronic care patients are followed (e.g., palliative care, rehabilitation, psychiatry, geriatrics, family medicine) and the biopsychosocial model of practice is forefront.

\section{Conclusions}

Findings from this study draw our attention to how expertise may be challenged by context and how residents exposed to an unfamiliar outpatient chronic care setting may adopt a more simplistic view of relevance to equate it with such defined principles such as data organization. Thus, clinical teachers in these settings should have a heightened awareness of residents' potential difficulties with applying their rhetorical knowledge of relevance to the OCP. Our results suggest that a resident who might be expected to have mastered OCP skills on core paediatrics rotations may not perform the OCP fluently in a different clinical context. With this understanding, appropriate supportive teaching strategies can be implemented. As the OCP is considered by medical training regulatory bodies (RCPSC in Canada, ACGME in the U.S.) as a core competency skill that must be achieved for successful practice as an independent clinician, the importance of faculty support and teaching around OCP skills is particularly relevant in helping residents achieve and adapt this essential skill.

\section{Study limitations}

The design of this study places some constraints on the insights we can gain from it. The study captured a relatively small sample size and repeated observations of participants was limited in some cases, confining our insights to those recurrent patterns that emerged within this data set. As with any qualitative study, we do not make claims to empirical generalisability but rather raise issues whose transferability can be explored in further research in related or contrasting environments. The strength of this work lies in its conceptual generalisability and ability to explore resident OCPs and related teaching exchanges in situ, capturing them as they are delivered, and subsequently talking to residents and staff to understand the significance of the OCP for their learning experiences in this setting. Finally, we do not know whether these particular residents were expert case presenters in the acute, core pediatric care settings. Their stage of training would suggest that a high level of fluency in case presentation would have been achieved in the clinical contexts with which they were familiar. Future research could compare OCP performance across clinical contexts to further our insight into the influence of context on OCP expertise.

\section{Conflict of Interest}

The authors declare that they have no conflict of interest.

\section{References}

1. Spafford MM, Schryer CF, Mian M, Lingard L. Look who's talking teaching and learning using the genre of medical case presentations. J Bus Tech Commun. 2006;20(2):121-58.

2. Lingard LA, Haber RJ. What do we mean by "relevance"? A clinical and rhetorical definition with implications for teaching and learning the casepresentation format. Acad Med. 1999;74(10 Suppl):S124-7.

3. Haber RJ, Lingard LA. Learning oral presentation skills: a rhetorical analysis with pedagogical and professional implications. J Gen Intern Med. 2001;16(5):308-14.

4. Green EH, Durning SJ, DeCherrie L, Fagan MJ, Sharpe B, Hershman W. Expectations for oral case presentations for clinical clerks: opinions of internal medicine clerkship directors. J Gen Intern Med. 2009;24(3):370-3.

5. Aziz N, Goldszmidt M, Lingard LA. Taking a detour: positive and negative impacts of supervisor interruptions during admission case review. Acad Med. 2012; 87(10):1382-1388.

6. Holmboe ES, Ward DS, Reznick RK, Katsufrakis PJ, Leslie KM, Patel VL, et al. Faculty development in assessment: the missing link in competencybased medical education. Acad Med. 2011;86(4):460-7.

7. Frank JR. The CanMEDs 2005 Physician competency framework: better standards, better physicians, better care. Ottawa: Royal College of Physicians and Surgeons of Canada; 2005.

8. The Accreditation Council for Graduate Medical Education and the American Board of Pediatrics. Pediatric milestones working group. [cited 13 June 2012]; Available from: https://www.acgme.org/acWebsite/RRC _320/320_PedsMilestonesProject.pdf.

9. Yang G, Chin R. Assessment of teacher interruptions on learners during oral case presentations. Acad Emerg Med. 2007;14(6):521-5.

10. Papp KK, Wolpaw T. Clinical reasoning in resident case presentations. Med Educ. 2010;44(5):512.

11. Kihm JT, Brown JT, Divine GW, Linzer M. Quantitative analysis of the outpatient oral case presentation: piloting a method. J Gen Intern Med. 1991;6(3):233-6.

12. Boyatzis RE. Transforming qualitative information: thematic analysis and code development. Thousand Oaks: Sage Publications; 1998.

13. Morse JM. The significance of saturation. Qual Health Res. 1995;5(2):147-9.

14. Fox RC. Training for uncertainty. In: Merton RK, Reader GG, Kendall PL, editors. The student-physician; introductory studies in the sociology of medical education. Cambridge: Harvard University Press; 1957. p. 207-41.

15. Lingard L, Garwood K, Schryer CF, Spafford MM. A certain art of uncertainty: case presentation and the development of professional identity. Soc Sci Med. 2003;56(3):603-16.

16. Geller G, Faden RR, Levine DM. Tolerance for ambiguity among medical students: implications for their selection, training and practice. Soc Sci Med. 1990;31(5):619-24.

17. Green EH, Hershman W, DeCherrie L, Greenwald J, Torres-Finnerty N, Wahi-Gururaj S. Developing and implementing universal guidelines for oral patient presentation skills. Teach Learn Med. 2005;17(3):263-7.

18. Klos M, Reuler JB, Nardone DA, Girard DE. An evaluation of trainee performance in the case presentation. J Med Educ. 1983;58(5):432-4. 
19. Chang RW, Bordage G, Connell KJ. The importance of early problem representation during case presentations. Acad Med. 1998;73(10 Suppl):S109-11.

20. Nendaz MR, Junod AF, Vu NV, Bordage G. Eliciting and displaying diagnostic reasoning during educational rounds in internal medicine: who learns from whom? Acad Med.1998;73(10 Suppl):S54-6.

21. Pangaro L. A new vocabulary and other innovations for improving descriptive in-training evaluations. Acad Med. 1999;74(11):1203-7.

22. Green EH, DeCherrie L, Fagan MJ, Sharpe BA, Hershman W. The oral case presentation: what internal medicine clinician-teachers expect from clinical clerks. Teach Learn Med. 2011;23(1):58-61.

23. Jackson JL, O'Malley PG, Salerno SM, Kroenke K. The teacher and learner interactive assessment system (TeLIAS): a new tool to assess teaching behaviors in the ambulatory setting. Teach Learn Med. 2002;14(4):249-56.

24. Irby DM. What clinical teachers in medicine need to know. Acad Med. 1994;69(5):333-42.

25. Wiese J, Varosy P, Tierney L. Improving oral presentation skills with a clinical reasoning curriculum: a prospective controlled study. Am J Med. 2002;112(3):212-8.

26. Kogan JR, Conforti LN, Bernabeo EC, Durning SJ, Hauer KE, Holmboe ES. Faculty staff perceptions of feedback to residents after direct observation of clinical skills. Med Educ. 2012;46(2):201-15. 